Bahr L, Theorell K. Motor impairments in children with epilepsy treated with carbamazepine. Acta Paediatr April 1997;86:372-376). (Respond: Dr G Braathen, Department of Paediatrics, Huddinge University Hospital, S-141 86 Huddinge, Sweden).

COMMENT. Fine motor coordination impairments in children with epilepsy treated with carbamazepine may be explained by effects of the antiepileptic drug.

Cerebellar adverse effects of carbamazepine are reported in 9 (35\%) of 26 young adults with chronic focal epilepsy treated at the Klinik Mara 1, Epilepsie-Zentrum Bethel, Bielefeld, Germany. (Specht U, May TW, Rohde M et al. Cerebellar atrophy decreases the threshold of carbamazepine toxicity in patients with chronic focal epilepsy. Arch Neurol April 1997;54:427-431). Serum concentrations of $\mathrm{CBZ}$ at onset of ataxic side-effects were related to occurrence of cerebellar atrophy on MRI. Patients showing cerebellar atrophy developed ataxia, nystagmus, and dizziness at lower CBZ levels than those without cerebellar atrophy.

\title{
EPILEPSY, ADHD, AND METHYLPHENIDATE
}

The effects of methylphenidate in thirty children, ages 6 to 16 , with epilepsy and ADHD were evaluated in a 4-month period at Shaare Zedek Medical Center, Hadassah-Hebrew University, Jerusalem, Israel. Using a double-blind, crossover design, patients received EEGs, AED level determinations, and continuous-performance tasks during treatment with AEDs only, and with the addition of methylphenidate (MPH), $0.3 \mathrm{mg} / \mathrm{kg}$ each morning, or placebo. Three of 5 children with seizures had exacerbation of attacks ( 3 to 7 attacks per week) while taking MPH, while 25 without seizures continued to be seizurefree. MPH improved ADHD symptoms and continuous-performance task scores. (Gross-Tsur V, Manor O, van der Meere J, Joseph A, Shalev RS. Epilepsy and attention deficit hyperactivity disorder: Is methylphenidate safe and effective? I Pediatr April 1997;130:670-674). (Reprints: Ruth Shalev MD, Pediatric Neurobehavioral Unit, Shaare Zedek Medical Center, POB 3235, Jerusalem, Israel).

COMMENT. Methylphenidate (MPH) may be a safe and effective treatment for ADHD complicating childhood epilepsy, provided that patients are receiving antiepileptic drugs when MPH is initiated and that seizures are under satisfactory control. Patients with persisting seizures despite AEDs may have an exacerbation with MPH. Existing studies do not address the problem of patients with ADHD in whom a predisposition to seizures may be overlooked before stimulant therapy is introduced. Furthermore, the effects of Ritalin ${ }^{\circledR}$ may be different from generic MPH.

Ritalin-induced seizures in two children with ADHD. Two boys, ages 8 and 11 years, were seen in the ADD Clinic, Division of Neurology, Children's Memorial Hospital, Chicago, with a history of convulsive seizures after Ritalin had been introduced for treatment of ADHD. With the addition of carbamazepine and withdrawal or reduction in dosage of Ritalin, seizures had not recurred. (Millichap JG, Swisher CN. Manuscript in preparation).

\section{TOURETTE SYNDROME}

\section{METABOLIC ANATOMY OF TOURETTE'S SYNDROME}

A Scaled Subprofile Statistical Model (SSM) of regional metabolic covariation was employed to identify functional brain networks in 10 Tourette 
syndrome (TS) patients (mean age, 41 years), with 10 normal volunteers as controls, in a study at North Shore University Hospital, Manhasset, New York. Quantitative fluorodeoxyglucose (FDG) and PET were used to calculate global and regional rates of glucose metabolism. While global glucose metabolic rates were normal in TS patients, SSM analysis identified bilateral metabolic increases in lateral premotor and supplementary motor cortex and midbrain, and decreases in caudate and thalamic metabolism. TS patients have a nonspecific pattern of increased motor cortical activity common to other hyperkinetic disorders, and a specific brain network involving reduced activity of limbic basal ganglia-thalamocortical projections. (Eidelberg D, Moeller JR, Antonini A et al. The metabolic anatomy of Tourette's syndrome. Neurology April 1997;48:927-934). (Reprints: Dr D Eidelberg, Movement Disorders Center, North Shore University Hospital, 444 Community Drive, Manhasset, New York, 11030).

COMMENT. Two independent brain networks are identified in patients with Tourette syndrome: 1) increased metabolic activity of motor cortical regions involved in execution of movement; and 2) decreased metabolic activity in basal ganglia-thalamic areas governing TS global scale severity.

\section{GENETICS OF TOURETTE SYNDROME}

Age at onset, age at diagnosis, and phenotypic expressions of TS were compared in the offspring of affected males and females among 437 first degree relatives of 57 probands studied at Academic Department of Psychiatry, University College London Medical School, London, UK. Maternally transmitted cases had an earlier age at onset, suggesting a genomic imprinting effect on the expression of TS. (Eapen V, O'Neill J, Gurling HMD, Robertson MM. Sex of parent transmission effect in Tourette's syndrome: Evidence for earlier age at onset in maternally transmitted cases suggests a genomic imprinting effect. Neurology April 1997;48:934-937). (Reprints: Dr V Eapen, Faculty of Medicine \& Health Sciences, United Arab Emirates University, PO Box 17666, Al Ain, United Arab Emirates).

COMMENT. Family history data on Tourette syndrome cases must be evaluated for maternal or paternal transmission. Mothers affected with TS are likely to have affected offspring with an earlier age at onset. Environmental factors, such as exposure to stress or cocaine, may be alternative explanations for this finding.

Developmental Basal Ganglia Syndrome. Failure to distinguish TS from other causes of DBGS may explain confusion in localizing the genetic defect in TS, determining prevalence, and assessing therapy, according to a report from the University of Rochester, NY. (Palumbo D, Maughan A, Kurlan R. Hypothesis III. Tourette syndrome is only one of several causes of developmental basal ganglia syndrome. Arch Neurol April 1997;54:475-483). Causes of the DBGS include primary (hereditary) tic disorders, obsessivecompulsive disorder, ADHD, Huntington disease, and secondary (symptomatic) autism, mental retardation, intrauterine drug exposure, perinatal asphyxia, encephalitis, head trauma, etc.

\section{STIMULANT THERAPY OF ADHD AND TOURETTE SYNDROME}

The effects of methylphenidate (MPH) and dextroamphetamine (DEX) on tic severity in 20 boys with ADHD and comorbid Tourette syndrome were investigated in a 9-week, placebo-controlled, double-blind crossover study at 